\title{
Étude des possibilités d'allègement du contrôle laitier officiel chez les caprins
}

\author{
N Bouloc 1, F Barillet, D Boichard 2, JP Sigwald 3, G Bridoux 4 \\ 1 INRA-SAGA, BP 27, 31326 Castanet Tolosan Cedex; \\ 2 INRA-SGQA, Domaine de Vivert, 78352 Jouy-en-Josas Cedex; \\ 3 ITOVIC, 149, rue de Bercy, 75595 Paris; \\ ${ }^{4}$ SAPERFEL, BP 312, 79009 Niort Cedex, France
}

(Reçu le 14 décembre 1990; accepté le 22 avril 1991)

\begin{abstract}
Résumé - Différentes options d'allègement du contrôle laitier officiel A30 (contrôle mensuel des 2 traites quotidiennes) basées sur l'espacement des contrôles ou sur le contrôle mensuel d'une seule des 2 traites quotidiennes sont simulées à partir de 4 échantillons de 307, 757, 6347 et 2275 chèvres. Pour toutes les méthodes d'allègement envisagées, la perte de précision sur le calcul des performances de lactation est comparable dans le cas des quantités de lait, de matière protéique et du taux protéique. Elle est moins élevée que dans le cas de la quantité de matière grasse et surtout du taux butyreux. La précision du contrôle laitier dépend plus du nombre de contrôles que du nombre de traites mesurées à chaque contrôle. Ainsi par rapport à la stratégie $A 30$, la méthode A42 (contrôle des 2 traites quotidiennes tous les 42 j environ) entraîne une perte de précision de $50 \%$ supérieure à la méthode préconisée dite AT30 (contrôle en alternance de la traite du matin et du soir). Dans ce dernier cas, par rapport à la méthode A30, la perte de précision sur l'estimation de la production de lait à $200 \mathrm{j}$ est de $2.3 \%$ et reste compatible avec la finalité génétique du contrôle laitier. Le léger biais généré par la nature du premier contrôle, réalisé à la traite du matin ou du soir, peut être maîtrisé par différentes méthodes d'ajustement. Si l'estimation de la quantité de lait quotidienne est meilleure à partir de la production de lait mesurée à la traite du matin plutôt que celle du soir, elle reste relativement imprécise et sa valorisation par l'appui technique se révèle plus délicate.
\end{abstract}

contrôle laitier / allègement / caprin

Summary - Simplification of the official milk record for goats. Simplified designs of the official A30 milk recording (monthly records of the 2 daily milkings) based on spacing of records or on 1 daily sampling every month were simulated on 4 data samples including 307, 757, 6347 and 2275 lactations. In this study, the A42 option (sampling every 42 d of the 2 daily milkings) is not very precise. The loss of precision compared to the A3O option in $\approx 7 \%$ for milk yield (table IV). The biais is about zero but influenced by the stage of the first control. All options with 1 daily sampling every month (AM30 morning sampling, AS30 afternoon sampling, AT30 alternate sampling) are more accurate than the A42 sampling. For estimation of milk production, the number of sample days per lactation rather than number of milkings measured per sample day limits accuracy. As regards the estimation of milk yield, the loss of accuracy is $\approx 2 \pm 3.5 \%$ (tables VI, VII) with 1 sampling a day every month. The AS30 option is always worse than the AM3O or the AT3O. The biais could be important if only 1 type of milking is sampled $(\approx 50 \mathrm{~kg}$ of milk; tables VI, VII). However, it could be controlled by additional information from the unsampled milking, such as milking intervals or milk production of the entire flock on the test-day. For milk yield, protein yield and protein content, consequences of simplified design are comparable and smaller than for fat yield and fat content where loss of precision is $>10 \%$ 
(tables $N, V I)$. These results indicate that the AT30 procedure may be suggested for goat milk recording. The loss of precision for the 2 selection criteria is minimal and compatible with the genetic aim of milk recording. The prediction of daily milk yield is easier with the milk yield measured for the morning milking than for the afternoon milking (table VI); however, it remains rather inaccurate, and its use as a management tool presents certain problems.

milk recording / simplification / goat

\section{INTRODUCTION}

Le contrôle laitier officiel a pour objet la mesure des productions laitières d'un animal, au cours de ses lactations successives, en vue de l'estimation de performances de production par lactation. Celles-ci sont utilisées dans le calcul d'indices de sélection pour l'évaluation génétique des reproducteurs. Le contrôle laitier est aussi valorisé par l'appui technique fourni à l'éleveur. L'efficacité de l'estimation des performances laitières dépend de la précision des mesures ponctuelles et successives de production, du mode de calcul de la lactation et de la méthodologie du contrôle laitier.

Dans l'espèce caprine la pratique actuelle du contrôle laitier officiel est, comme chez les bovins, basée sur le contrôle mensuel des productions quotidiennes. L'intérêt de l'allègement réside dans la diminution de son coût dont la charge, ramenée à la marge brute par femelle, est 3 à 4 fois plus élevée pour les caprins que pour les bovins. La réduction du nombre de passages du technicien de contrôle laitier est un facteur d'amélioration de la productivité du travail. Moins contraignant pour l'éleveur, le contrôle est aussi mieux toléré. Mais il ne peut être envisagé que si la perte de précision due à la diminution du nombre de mesures reste compatible avec une évaluation correcte des reproducteurs. De nombreux auteurs ont proposé des modalités d'allègement en soulignant que la réduction du nombre de traites contrôlées est préférable à l'espacement des contrôles (Mc Daniel, 1969; Munro, 1976; Barillet et al, 1987). La difficulté essentielle est alors l'estimation de la production quotidienne à partir des performances de la seule traite du soir ou du matin. Everett et Wadell (1970b) ont mis en évidence la supériorité des facteurs de correction multiplicatifs sur les facteurs additifs pour cette estimation.

Dans cette étude, plusieurs nypothèses d'allègement de la pratique actuelle ont été simulées en vue de comparer leurs acceptabilités et évaluer les possibilités de mise en applıcation dans l'espèce caprine.

\section{CONDITIONS EXPÉRIMENTALES}

\section{Données disponibles}

Les donnees analysées ont été recueillies dans le cadre d'une experimentation conduite conjointement par l'INRA (Institut national de la recherche agronomique) et I'ITOVIC (Institut technique de l'élevage ovin et caprin).

En 1987, la Station de Testage de Moissac (Lozère) et 6 élevages du Poitou, puis en 1988 le Syndicat de contrôle laitier des Deux-Sevres ont collaboré à l'étude. Les mesures sont prises dans le cadre du contrôle laitier mensuel sauf à Moissac où il est bimensuel. Dans tous les cas, les informations normalement recueilies, date de mise-bas et de contròle, lait produit le jour du contrôle (QL), taux butyreux (TB) et protéique (TP) sont disponibles. Les quantités de lait du 
soir (QLS) et du matin (QLM) sont connues dans les 3 cas.

Le fichier 1 (Moissac) concerne 307 chèvres, 3354 contrôles élémentaires et dispose des taux protéiques des laits du soir (TPS) et du matin (TPM).

Le fichier 2 ( 6 élevages du Poitou) concerne 757 chèvres avec 5171 contrôles ponctuels soit 7 à 8 contrôles par troupeaux. II dispose des richesses du lait du soir (TBS, TPS) et du matin (TBM, TPM) et des $h$ de début et fin de traite.

Le fichier 3 concerne 660 élevages, 57914 chèvres soit 203004 contrôles élémentaires. Seul un nombre limité de troupeaux dispose des quantités du tank mesurées à l'issue des traites du soir et du matin ou des $\mathrm{h}$ de début et de fin de traite pour l'ensemble des contrôles laitiers de la campagne. Cela a conduit à l'organisation en 2 fichiers d'étude où sont possibles les calculs des performances de lactation :

- un fichier «horaire" (fichier 3a) combinant l'information élémentaire du contrôle laitier et les horaires de traite (41 390 enregistrements élémentaires concernant 6347 chèvres réparties dans 112 élevages),

- un fichier "tank» (fichier $3 b$ ) combinant l'information élémentaire du contrôle laitier et la production du troupeau le jour du contrôle (14 266 enregistrements élémentaires concernant 2275 chèvres de 41 troupeaux).
Les caractéristiques des fichiers d'étude sont résumées dans le tableau I.

\section{Hypothèses d'allègement envisagées}

\section{Modalités de passage du contrôleur (tableau II)}

La pratique actuelle du contrôle laitier officiel caprin (modalité $\mathrm{A} 30$ ) la plus fréquente $(80 \%$ des chèvres contrôlées) est basée sur le contrôle mensuel des 2 traites quotidiennes de tous les animaux traits durant toute leur lactation. Alléger ce contrôle en réduisant le nombre de passages du contrôleur dans l'élevage peut prendre plusieurs modalités.

\section{Réduire le nombre de contrôles et adopter} un rythme irrégulier

Cette modalité privilégie certaines parties de la lactation. Cette stratégie n'a pas été envisagée dans cette étude car elle suppose une conduite très saisonnée des animaux. De plus, d'après Anderson et al (1989), lorsque le nombre de contrôle au cours de la campagne est réduit $(<10)$, celle-ci n'est pas satisfaisante.

Tableau I. Fichiers d'étude.

\begin{tabular}{|c|c|c|c|c|c|c|}
\hline \multirow[t]{2}{*}{ Fichier } & \multicolumn{3}{|c|}{ Effectifs } & \multirow[t]{2}{*}{ Périodicité } & \multirow{2}{*}{$\begin{array}{l}\text { Quantités } \\
\text { contrôlées }\end{array}$} & \multirow{2}{*}{$\begin{array}{l}\text { Informations } \\
\text { complémentaires }\end{array}$} \\
\hline & troupeaux & femelles & contrôles & & & \\
\hline 1 & 1 & 307 & 3354 & $14 j$ & $\begin{array}{l}\text { Lait, TP } \\
\text { matin, soir }\end{array}$ & \\
\hline 2 & 6 & 757 & 5171 & $30 \mathrm{j}$ & $\begin{array}{l}\text { Lait, TB, TP } \\
\text { matin, soir }\end{array}$ & horaires \\
\hline $3 a$ & 112 & 6347 & 41390 & $30 \mathrm{j}$ & $\begin{array}{l}\text { Lait } \\
\text { matin, soir }\end{array}$ & horaires \\
\hline $3 b$ & 41 & 2275 & 14266 & $30 \mathrm{j}$ & $\begin{array}{l}\text { TB, TP } \\
\text { quotidien }\end{array}$ & $\begin{array}{l}\text { production totale } \\
\text { du troupeau }\end{array}$ \\
\hline
\end{tabular}

1 station expérimentale de Moissac (48); 2 élevages du Poitou; $3 a$ et 3b syndicat de contrôle laitier des DeuxSevres. 
Tableau II. Type de contrôles.

$\begin{array}{ccc}\text { Nom } & \begin{array}{c}\text { Périodicité de passage } \\ \text { du contrôleur }\end{array} & \text { Traites contrôlées }\end{array}$

$\begin{array}{lll}\text { A14 } & 14 j & \text { matin et soir } \\ \text { A30* } & 30 j & \text { matin et soir } \\ \text { A42 } & 42 j & \text { matin et soir } \\ \text { A60 } & 60 j & \text { matin et soir } \\ \text { AT30 } & 30 j & \text { en alternance matin et soir } \\ \text { AM30 } & 30 j & \text { matin } \\ \text { AS30 } & 30 j & \text { soir }\end{array}$

- méthode actuelle, de référence.

Garder un rythme régulier des contrôles mais les espacer

La méthodologie $\mathrm{A} 42$, pratiquée sur $20 \%$ des chèvres au contrôle laitier, suppose un contrôle tous les $42 \mathrm{j}$ environ. Cette hypothèse est simulée à partir des données du fichier 1 où les contrôles ont été réalisés tous les 14 j avec 16 contrôles au maximum par chèvre et 8,8 en moyenne. Les dispositifs basés sur une périodicité de 28 et $42 \mathrm{j}$ sont simulés en supprimant 1 contrôle sur 2 ou 2 contrôles sur 3.

Garder un rythme régulier mensuel mais ne contrôler qu'une seule des 2 traites quotidiennes

Plusieurs protocoles de passage du contrôleur sont envisagés à partir des données des fichiers 2, $3 a$ et $3 b$ :
- le contrôle ne porte que sur la traite du matin (AM30);

- le contrôle ne porte que sur la traite du soir (AS30);

- le contrôle intervient dans l'élevage en alternance (AT30) à la traite du soir (mois $M$ et à la traite du matin (mois $N+1$ ).

\section{Modalités de calcul des performances quotidiennes (tableau III)}

Dans ce dernier cas, préalablement au calcul des performances de lactation, il faut estimer la production et les taux journaliers.

Dans le cas des quantités, une première solution consiste à doubler la production enregistrée à la traite contrôlée. Cette hypothèse est simulée sur les fichiers 2, $3 a$ et $3 b$.

Tableau III. Mode de calcul de la production quotidienne de lait à partir de la production à une traite.
$\times 2 \quad$ Production de la traite contrôlée multipliée par 2
int Correction pour l'intervalle entre les traites du soir et du matin (formule de régression)
tank Correction par rapport à la production totale du troupeau mesurée le jour du contrôle. 
Une deuxième méthode consiste à ajuster la part de production quotidienne assurée à la traite contrôlée par l'intervalle entre traites. Soit $Q L_{i j}$ et $Q L_{i j k}$ les quantités de lait totales et de la traite $k$ respectivement, produit dans l'élevage $i$ lors du contrôle $j$. Le rapport $R_{i j}$ est alors défini par $Q L_{i j}=A_{i j} \cdot Q L_{i j k}$ et estimé par régression linéaire sur l'intervalle entre la traite du soir et du matin $\left(I N T_{i j}\right)$ à partir de 1652 contrôles dans 345 élevages : matin

$$
R_{i j}=0,468+0,131 \cdot I N T_{i j} \text { pour la traite du soir; }
$$

$R_{i j}=3,283-0,108 \cdot / N T_{i j}$ pour la traite du

Sur l'échantillon, laits du soir et du matin sont égaux pour un intervalle soir-matin de $11 \mathrm{~h} 45$. Avec un intervalle de $12 \mathrm{~h}$, le lait du matin représente environ $51 \%$ de la production totale de la journée. Ce type d'ajustement est simulé à partir des données des fichiers 2 et $3 a$.

Une troisième approche prend en compte la fraction de la production quotidienne du troupeau obtenue à l'issue de la traite contrôlée.

$$
Q L_{i j}=K_{k} \cdot Q L_{k i j} \text { avec } K_{k}=\left(T M_{i j}+T S_{i j}\right) / Q L_{k i j}
$$

où $T M_{i j}$ et $T S_{i j}$ sont les quantités de lait mesurées dans le tank à l'issue des traites du matin et du soir, $Q L_{k i j}$ la somme des quantités de lait mesurées à la traite $k$ contrôlée (matin ou soir) à la date $j$ dans l'élevage $i$.

Elle est envisagée à partir des données des fichiers 2 et 3b. Dans le cas du fichier 2, l'information du tank n'existant pas, elle est estimée par la somme des quantités individuelles de lait contrôlées.

L'application des ajustements intervalle de traite et tank est fondée sur l'hypothèse que la situation moyenne de l'élevage traduit les variations soir matin de productions individuelles. Cela suppose que toutes les chèvres traites sont contrôlées pour la mesure du lait du tank, que le passage du contrôleur ne modifie pas sensiblement les horaires de traites.

\section{Critères d'évaluation de l'efficacité des methodes}

En l'absence d'informations plus précises, la production laitière calculée à partir des résultats du contrôle A30 est prise comme référence. Elle représente déjà une mesure indirecte de la production vraie. En élevage bovin, avec 8 contrôles en moyenne, le niveau de précision atteint $(97 \%$ pour la quantité de lait, $96,5 \%$ pour la quantité de matière grasse, $95 \%$ pour le taux butyreux, Mc Daniel, 1969 et Anderson et al, 1989) a permis de reconnaître officiellement cette production calculée pour l'évaluation des reproducteurs. Dans cette étude, la production de référence est la lactation totale, calculée avec un minimum de 2 contrôles enregistrés dans le cas des fichiers 1 et 2, la lactation tronquée à 200 j pour les fichiers $3 a$ et $3 b$.

Cette étude de l'allègement du contrôle laitier se limite à la recherche de solutions techniques acceptables par le sélectionneur et par l'éleveur. Une bonne efficacité du point de vue phénotypique, garante d'une bonne efficacité du point de vue génétique, est absolument recherchée. L'efficacité phénotypique de chaque méthode allégée envisagée est appréciée par la comparaison de la mesure de référence $(Y)$ et de la mesure $(X)$ estimée après allègement (moyenne et écart type) et le calcul de l'erreur systématique ou biais ( $D=Y-X)$. Le calcul du coefficient de corrélation intra-élevage $R$ entre $Y$ et $X$ traduit le classement des individus. Le coefficient de régression (b) de $Y$ en $X(Y=a+b \cdot X+E)$ indique la nature de l'erreur. Si b est égal à 1, le biais est indépendant de l'erreur aléatoire $E$ et seule la perte de précision $\left(1-R^{2}=\sigma^{2} E^{\prime} \sigma^{2} Y\right)$ affecte le classement des individus. Si b est différent de 1, le biais qui n'est plus indépendant du niveau de production et l'erreur aléatoire concourent simultanément à diminuer l'efficacité de la méthode allégée.

\section{L'ALLĖGEMENT PAR ESPACEMENT DES CONTROOLES}

La comparaison des méthodologies de contrôle laitier pratiqué tous les 30 ou 42 j est conduite à partir des données du fichier 1 (tableau IV).

\section{Précision}

Entre 14 et 28 j, la perte de précision est relativement faible : de l'ordre de $2 \%$ pour 
le lait et le TP, de $3 \%$ pour la quantité de matière protéique. De même, l'écart type de l'erreur observée entre les productions estimées à partir d'un contrôle bimensuel ou mensuel est assez peu élevée, de l'ordre de $5 \%$ de la moyenne pour les quantités de lait et de matière protéique, de l'ordre de $1 \%$ pour le taux protéique.

Par contre, les résultats obtenus en passant d'un contrôle mensuel à 1 contrôle tous les $42 \mathrm{j}$ sont nettement moins bons (tableau IV). La perte de précision se situe autour de $7 \%$ pour le lait, de $8 \%$ pour la QMP, de $5 \%$ pour le TP. Les écarts types de l'erreur sont 2 fois plus élevés que dans le cas précédent. Ce phénomène s'explique par le fait que l'allègement est d'autant moins précis que le nombre de mesures de référence est faible.

Mc Daniel (1969), dans sa revue bibliographique, indique que les erreurs commises sur l'estimation de la production laitière augmentent fortement lorsqu'on envisage un contrôle de type $A 60$ plutôt qu'un contrôle de type A30. Dans le cas de la quantité de lait, le pourcentage d'erreur est de $3,8 \%$ et $2,5 \%$ respectivement; $22 \%$ des lactations s'écartent de plus de $5 \%$ de la production vraie avec un contrôle A60 contre $7 \%$ dans le cas d'un contrôle A30. Une part importante des erreurs est imputable au stade de lactation auquel intervient le premier contrôle.

\section{Biais}

D'une manière générale, avec un contrôle tous les $42 \mathrm{j}$, la surestimation des quantités de lait et de matière protéique est nette (tableau IV). Comme le souligne Mc Daniel (1969), une part de l'erreur est imputable au stade de lactation auquel intervient le premier contrôle.

Toutefois, la pente (b) de la droite de régression de la production de référence sur la production estimée à partir d'un contrôle moins fréquent est pour les 3 caractères analysés proche de 1 : si le biais n'est donc pas négligeable il ne dépend pas du niveau de production.

Tableau IV. Efficacité des méthodes allégées de contrôle laitier; conséquences sur le calcul des performances laitières sur les données du fichier 1 .
Lait $(\mathrm{kg})$
$Q M P(k g)$
$T P(g / k g)$

Comparaison des méthodes $\mathrm{A} 30$ et $\mathrm{A} 42(\mathrm{n}=224)$

$\begin{array}{lccc}Y_{A 30} & 482 \pm 201 & 13,0 \pm 5,2 & 27,2 \pm 2,2 \\ X_{A 42} & 525 \pm 191 & 14,1 \pm 5,0 & 27,2 \pm 2,2 \\ D=Y_{A 30}-X_{A 42} & -42 \pm 55 & -1,1 \pm 1,4 & 0,05 \pm 0,5 \\ \sigma_{E} & 46 & 1,2 & 0,5\end{array}$

Comparaison des méthodes A30 et AT30 $(n=263)$

$\begin{array}{lccr}Y_{A 30} & 487 \pm 197 & 13,1 \pm 5,1 & 27,2 \pm 2,2 \\ X_{A T 30} & 500 \pm 206 & 13,4 \pm 5,3 & 27,2 \pm 2,3 \\ D=Y_{A 30}-X_{A T 30} & -13 \pm 21 & -0,4 \pm 0,6 & -0,04 \pm 0,3 \\ \sigma_{E} & 21 & 0,6 & 0,3\end{array}$




\section{L'ALLĖGEMENT PAR SUPPRESSION DU CONTRÓLE D'UNE DES DEUX TRAITES QUOTIDIENNES}

\section{Prédiction de la production quotidienne}

Dans le cas simple où on multiplie par 2 la production de lait mesurée, l'estimation de la production quotidienne reste relativement imprécise, les biais moyens observés sont élevés (tableau V), de signe opposé par construction, selon que l'on travaille à partir de la traite du matin ou du soir. La tendance est à la surestimation à partir du lait du matin. En effet, dans notre cas, la production du matin représente en moyenne $52 \%$ du lait quotidien. Dans un tiers des cas, les biais constatés s'écartent de plus de $10 \%$ de la production du jour.
Du point de vue de la richesse du lait, la situation est différente. Le TP varie peu entre le soir et le matin. La quantité de matière protéique se comporte, de ce fait, de manière identique à la quantité de lait. Les variations du TB entre le soir et le matin sont plus importantes; sur les données du fichier 2, le TB du matin surpasse celui du soir de $27 \%$ en moyenne. Les variations individuelles sont importantes et difficilement prévisibles. Par suite, l'estimation du TB quotidien à partir de l'information du taux à une seule traite présente de réelles difficultés.

La mise en place d'un ajustement parvient à annuler le biais moyen pour la prédiction de la quantité de lait. Cependant, dans environ $20 \%$ des cas le biais représente plus de $10 \%$ de la production quotidienne (18 et $23 \%$ des cas à partir de la

Tableau V. Prédiction de la production de lait quotidienne (QL) à partir de la production enregistrée à la truie du matin (QLM) ou du soir (QLS).

\begin{tabular}{|c|c|c|c|c|c|c|}
\hline \multirow[t]{2}{*}{ Mode de calcul } & \multicolumn{3}{|c|}{$x^{2}$} & \multicolumn{3}{|c|}{ Int } \\
\hline & $\begin{array}{c}\text { moyenne } \\
(\mathrm{kg})\end{array}$ & répétabilité & $\begin{array}{l}\text { écart type } \\
\text { d'erreur } \\
(\mathrm{kg})\end{array}$ & $\begin{array}{c}\text { moyenne } \\
(\mathrm{kg})\end{array}$ & répétabilité & $\begin{array}{l}\text { écart type } \\
\text { d'erreur } \\
(\mathrm{kg})\end{array}$ \\
\hline \multicolumn{7}{|c|}{$\begin{array}{l}\text { Fichier } 3 \mathrm{a} \\
\text { Production quotidienne mesurée }(\mathrm{Y})\end{array}$} \\
\hline \multicolumn{7}{|c|}{ Production estimée $(X)$} \\
\hline matin & $2,88 \pm 1,16$ & 0,62 & 0,27 & $2,74 \pm 1,10$ & 0,62 & 0,27 \\
\hline $\begin{array}{l}\text { soir } \\
\text { Ecart }(Y-X)\end{array}$ & $2,58 \pm 1,03$ & 0,60 & 0,30 & $2,76 \pm 1,10$ & 0,60 & 0,30 \\
\hline $\begin{array}{l}\text { matin } \\
\text { soir }\end{array}$ & $\begin{array}{r}-0,15 \pm 0,25 \\
0,15 \pm 0,25\end{array}$ & $\begin{array}{l}0,07 \\
0,07\end{array}$ & & $\begin{array}{l}-0,01 \pm 0,21 \\
-0,03 \pm 0,24\end{array}$ & $\begin{array}{l}0,03 \\
0,03\end{array}$ & \\
\hline \multicolumn{7}{|c|}{$\begin{array}{l}\text { Fichier } 3 b \\
\text { Production quotidienne mesurée }(Y)\end{array}$} \\
\hline \multicolumn{7}{|c|}{ Production estimée $(X)$} \\
\hline matin & $2,92 \pm 1,13$ & 0,64 & 0,27 & $2,79 \pm 1,07$ & 0,64 & 0,26 \\
\hline $\begin{array}{l}\text { soir } \\
\text { Ecart }(Y-X)\end{array}$ & $2,61 \pm 0,99$ & 0,61 & 0,30 & $2,79 \pm 1,07$ & 0,61 & 0,29 \\
\hline matin & $-0,15=0,25$ & 0,08 & & $-0,02 \pm 0,22$ & 0,05 & \\
\hline soir & $0,15 \pm 0,25$ & 0,08 & & $-0,02 \pm 0,24$ & 0,04 & \\
\hline
\end{tabular}


production du matin et du soir respectivement). Tout comme chez Schaeffer et Rennie (1976) et Lee et Wardrop (1984), les estimations sont plus efficaces à partir du contrôle du matin qu'à partir de celui du soir (précision plus élevée $93,5 \%$ vs $92 \%$ et écarts importants moins fréquents). La répétabilité de la production de lait est d'ailleurs légèrement plus élevée le matin. Les cas d'écarts anormaux concernent environ $20 \%$ des chèvres contrôlées à un contrôle donné. Ils se produisent sur l'ensemble de la campagne et à tous les stades de lactation. Ils affectent toutes les chèvres, quel que soit leur numéro de lactation, en moyenne 0,7 fois pour 4,1 contrôles connus. D'autre part, la répétabilité des écarts avec la production vraie est nulle. II semble donc que les difficultés d'estimation surviennent de façon aléatoire : elles semblent plutôt associées à des rapports de production individuelle soir/matin "hors norme".

Plusieurs causes sont possibles pour expliquer ces difficultés d'estimation : ordre de passage à la traite très différent entre les 2 traites, traite incomplète à la traite contrôlée ou à la précédente, erreur dans la transmission des données élémentaires. Comme le signalent Smith et Pearson (1981) et Lee et Wardrop (1984) la variabilité de la production à une traite est supérieure à celle de 2 traites successives cumulées.

\section{Calcul de lactation}

Le fichier 2 permet de comparer (tableau VI) les diverses méthodologies de contrôle laitier pour les 5 caractères habituellement calculés (quantités de lait, de matière grasse (QMG) de matière protéique (QMP), taux butyreux et protéique). Le cas de l'application d'une correction tenant compte de la quantité de lait du troupeau le jour du contrôle est simulée en calculant le lait du troupeau à partir de la somme des laits individuels mesurés.

Les fichiers $3 a$ et $3 b$ permettent de comparer les diverses méthodologies de contrôle laitier utilisant ou non des informations supplémentaires (horaires de traites ou lait de troupeau mesuré le jour du contrôle) dans les conditions prévues de leur utilisation (tableau VII). Seul, le cas du lait peut être étudié étant la seule variable pour laquelle les informations soir et matin sont disponibles.

\section{Précision}

Le classement des différentes méthodes envisagées est identique pour tous les caractères. Dans tous les cas, les protocoles imaginés à partir de la seule traite du soir (AS30) constituent l'alternative la moins bonne : perte de précision supérieure d'un tiers aux autres stratégies. La méthodologie AT30 apparaît légèrement plus précise que la stratégie $A M 30$ (perte de précision réduite de 10 à $15 \%$ ) pour le calcul de la production de lait à $200 \mathrm{j}$.

Le niveau de la perte de précision imputable à l'adoption de la stratégie AT sur le calcul des performances de lactation dépend de la nature du caractère. D'une manière générale, les quantités de lait et de matière protéique, ainsi que le taux protéique, sont les variables sur lesquelles on perd le moins de précision $(1,5$ à $2,5 \%)$. À l'opposé, le taux butyreux est le caractère le plus difficile à estimer. La précision reste inférieure à $90 \%$. La matière grasse se comporte de façon intermédiaire avec dans tous les cas une perte de précision de l'ordre de 3,5\%.

L'introduction d'un ajustement montre la possibilité de gain de précision variable selon le caractère considéré. Dans le cas de la quantité de lait, la mise en place d'un 
Tableau VI. Efficacité des méthodes allégées du contrôle laitier. Conséquence sur le calcul des performances laitières sur les données du fichier 2.

\begin{tabular}{|c|c|c|c|c|c|c|c|c|c|c|c|}
\hline Ajuste & ment & $\gamma$ & & $A M 30$ & & & $A S 30$ & & & AT3O & \\
\hline & & & $x$ & $Y-X$ & $\sigma_{E}$ & $x$ & $Y-X$ & $\sigma_{E}$ & $x$ & $Y-X$ & $\sigma_{E}$ \\
\hline $\begin{array}{l}\text { Lait } \\
(\mathrm{kg})\end{array}$ & $\begin{array}{l}x^{2} \\
\text { int } \\
\operatorname{tank}\end{array}$ & $\begin{array}{r}631 \\
\pm 263\end{array}$ & $\begin{array}{r}702 \\
+287 \\
638 \\
+263 \\
631 \\
+265\end{array}$ & $\begin{array}{r}-72 \\
\pm 63 \\
-7 \\
\pm 24 \\
0,1 \\
\pm 21\end{array}$ & $\begin{array}{l}34 \\
30 \\
30\end{array}$ & $\begin{array}{r}559 \\
+253 \\
622 \\
\pm 272 \\
631 \\
+263\end{array}$ & $\begin{array}{r}72 \\
\pm 63 \\
9 \\
\pm 42 \\
-0 \\
\pm 27\end{array}$ & $\begin{array}{l}41 \\
38 \\
37\end{array}$ & $\begin{array}{r}623 \\
\pm 260 \\
630 \\
+268 \\
631 \\
\pm 263\end{array}$ & $\begin{array}{r}7 \\
\pm 27 \\
0 \\
\pm 27 \\
0 \\
\pm 22\end{array}$ & 32 \\
\hline $\begin{array}{l}\mathrm{QMP} \\
(\mathrm{kg})\end{array}$ & $\begin{array}{l}x^{2} \\
\text { int } \\
\text { tank }\end{array}$ & $\begin{array}{r}16,6 \\
\pm 6,6\end{array}$ & $\begin{array}{r}18,4 \\
\pm 7,1 \\
16,7 \\
\pm 6,6 \\
16,5 \\
\pm 6,6\end{array}$ & $\begin{array}{r}-1,8 \\
\pm 1,6 \\
-0,1 \\
\pm 0,6 \\
0,1 \\
\pm 0,6\end{array}$ & $\begin{array}{l}0,9 \\
0,8 \\
0,8\end{array}$ & $\begin{array}{r}14,9 \\
\pm 6,5 \\
16,5 \\
\pm 6,9 \\
16,8 \\
\pm 6,7\end{array}$ & $\begin{array}{r}1,8 \\
\pm 1,6 \\
0,1 \\
\pm 1,1 \\
-0,1 \\
\pm 0,7\end{array}$ & $\begin{array}{l}1,0 \\
0,9 \\
0,9\end{array}$ & $\begin{array}{r}16,4 \\
\pm 6,6 \\
16,6 \\
\pm 6,8 \\
16,7 \\
\pm 6,7\end{array}$ & $\begin{array}{r}0,2 \\
\pm 0,7 \\
0,0 \\
\pm 0,7 \\
0,0 \\
\pm 0,6\end{array}$ & 0,8 \\
\hline $\begin{array}{l}\mathrm{TP} \\
(\mathrm{g} / \mathrm{kg})\end{array}$ & $\begin{array}{l}x^{2} \\
\text { int } \\
\text { tank }\end{array}$ & $\begin{array}{r}26,7 \\
\pm 2,4\end{array}$ & $\begin{array}{r}26,6 \\
\pm 2,5 \\
26,6 \\
\pm 2,5 \\
26,7 \\
\pm 2,5\end{array}$ & $\begin{array}{r}0,1 \\
\pm 0,3 \\
0,1 \\
\pm 0,3 \\
0,1 \\
\pm 0,3\end{array}$ & $\begin{array}{l}0,3 \\
0,3 \\
0,3\end{array}$ & $\begin{array}{r}26,7 \\
\pm 2,4 \\
26,9 \\
\pm 2,4 \\
26,8 \\
\pm 2,4\end{array}$ & $\begin{array}{l}-0,2 \\
\pm 0,4 \\
-0,2 \\
\pm 0,4 \\
-0,2 \\
\pm 0,4\end{array}$ & $\begin{array}{l}0,4 \\
0,4 \\
0,4\end{array}$ & $\begin{array}{r}26,9 \\
\pm 2,4 \\
26,7 \\
\pm 2,4 \\
26,9 \\
\pm 2,4\end{array}$ & $\begin{aligned} & 0,01 \\
\pm & 0,3 \\
- & 0.0 \\
\pm & 0,3 \\
- & 0,02 \\
\pm & 0,3\end{aligned}$ & 0,3 \\
\hline $\begin{array}{l}\text { QMG } \\
\text { (kg) }\end{array}$ & $\begin{array}{l}x^{2} \\
\text { int } \\
\text { tank }\end{array}$ & $\begin{array}{r}19,8 \\
\pm 7,9\end{array}$ & $\begin{array}{r}20,0 \\
\pm 7,8 \\
18,2 \\
\pm 7,2 \\
18,0 \\
\pm 7,3\end{array}$ & $\begin{array}{r}-0,2 \\
\pm 1,3 \\
1,6 \\
\pm 1,5 \\
1,8 \\
\pm 1,4\end{array}$ & $\begin{array}{l}1,5 \\
1,5 \\
1,5\end{array}$ & $\begin{array}{r}19,5 \\
\pm 8,2 \\
21,9 \\
\pm 8,9 \\
22,3 \\
\pm 8,9\end{array}$ & $\begin{array}{r}0,2 \\
\pm 1,5 \\
-2,1 \\
\pm 1,6 \\
-2,5 \\
\pm 2,1\end{array}$ & $\begin{array}{l}1,5 \\
1,5 \\
1,5\end{array}$ & $\begin{array}{r}19,7 \\
\pm 7,9 \\
20,2 \\
\pm 8,2 \\
20,3 \\
\pm 8,1\end{array}$ & $\begin{array}{r}0,1 \\
\pm 1,0 \\
-0,4 \\
\pm 1,1 \\
-0,5 \\
\pm 1,2\end{array}$ & 1,3 \\
\hline $\begin{array}{l}\text { TB } \\
(\mathrm{g} / \mathrm{kg})\end{array}$ & $\begin{array}{l}x^{2} \\
\text { int } \\
\text { tank }\end{array}$ & $\begin{array}{r}31,8 \\
\pm 3,8\end{array}$ & $\begin{array}{r}28,9 \\
\pm 3,5 \\
28,9 \\
\pm 3,7 \\
28,9 \\
\pm 3,8\end{array}$ & $\begin{array}{r}2,9 \\
\pm 1,7 \\
2,9 \\
\pm 1,7 \\
2,9 \\
\pm 1,7\end{array}$ & $\begin{array}{l}1,3 \\
1,3 \\
1,3\end{array}$ & $\begin{array}{r}35,9 \\
\pm 5,4 \\
35,9 \\
\pm 5,4 \\
35,9 \\
\pm 5,4\end{array}$ & $\begin{array}{l}-4,1 \\
\pm 3,1 \\
-4,1 \\
\pm 3,2 \\
-4,1 \\
\pm 3,1\end{array}$ & $\begin{array}{l}1,5 \\
1,5\end{array}$ & $\begin{array}{r}32,0 \\
\pm 4,0 \\
32,5 \\
\pm 4,2 \\
32,7 \\
\pm 4,3\end{array}$ & $\begin{array}{l}-0,2 \\
\pm 1,2 \\
-0,7 \\
\pm 1,5 \\
-0,9 \\
\pm 1,7\end{array}$ & 1,3 \\
\hline
\end{tabular}

AT30 : le premier contrôle de l'élevage est réalisé le matin.

ajustement peut réduire la perte de précision de $20 \%$ (tableau VI). En raison des variations minimes entre les taux protéiques du soir et du matin, le gain de précision acquis sur le lait avec un ajustement adéquat se répercute sur la quantité de matière protéique (gain de précision de l'ordre de 15\%). La précision de l'estima- tion du taux protéique de la lactation, calculé indirectement à partir de la quantité de lait et de matière protéique, est peu sensible aux ajustements réalisés.

L'application d'un ajustement sur le lait ne permet pas de gagner de la précision sur la quantité de matière grasse ou sur le taux butyreux. Les variations de richesse 
en matière grasse du lait entre le soir et le matin sont importantes et imprévisibles (Lee et Wardrop, 1984), rendant plus difficiles leurs corrections.

Les niveaux de précision atteints se traduisent par un écart type d'erreur qui représente $4-5 \%$ de la production totale dans le cas des quantités et du taux butyreux, $1 \%$ de la production de taux protéique. Le gain de précision acquis après application d'un ajustement se traduit par une légère diminution de l'écart type d'erreur.

L'efficacité de l'ajustement dépend aussi de l'information utilisée. Dans les conditions de recueil en ferme, telles qu'appliquées pour la constitution du fichier 3 , on n'observe aucun gain sensible pour l'estimation des productions en $200 \mathrm{j}$ (tableau VII). Les résultats observés dans le tableau VI et ceux relevés dans la bibliographie prouvent que l'on peut mieux faire (Everett et Wadell, 1970a; Schaeffer et Rennie, 1976; Wiggans, 1981; Delorenzo et Wiggans, 1986; Gabiña et al, 1986; Barillet, 1990).

\section{Biais}

Sans ajustements, les écarts $(5-6 \%$ de la production de référence pour la quantité de lait à 200 j) relevés avec les stratégies AM30 ou AS30 affectent les variables dans des sens opposés du fait du mode de calcul. Ainsi, avec un contrôle AM30, quantités de lait et de matière protéique sont surestimées, le taux butyreux sousestimé tandis que le taux protéique et la quantité de matière grasse sont peu biaisés. Avec un allègement de type AT30, les biais sont quasiment nuls. En contrôlant en alternance les traites du soir et du matin, les variations de production soir-matin sont compensées d'une fois sur l'autre lorsque le nombre de contrôles est suffisant. Toutefois, en raison du poids du premier contrôle dans le calcul des performances de lactation (Dickinson et Mc Daniel, 1970), on constate un léger écart entre les productions de référence et estimées selon que le premier contrôle est réalisé à la traite du matin ou du soir. Par exemple, celui-ci est de $6 \mathrm{~kg}$ pour la pro-

Tableau VII. Efficacité des méthodes allégées de contrôle laitier. Conséquence sur le calcul de la production de lait à 200 jours $(\mathrm{kg})$.

\begin{tabular}{|c|c|c|c|c|c|c|c|c|c|c|}
\hline \multirow[b]{2}{*}{ Ajustement } & \multirow[b]{2}{*}{$Y$} & \multicolumn{3}{|c|}{$A M 30$} & \multicolumn{3}{|c|}{ AS30 } & \multicolumn{3}{|c|}{ AT3O } \\
\hline & & $x$ & $\begin{array}{c}D \\
Y-X\end{array}$ & $\sigma_{E}$ & $x$ & $\underset{Y-X}{D}$ & $\sigma_{E}$ & $x$ & $\underset{Y-X}{D}$ & $\sigma_{E}$ \\
\hline $\begin{array}{ll}\text { Fichier } & x^{2} \\
3 a & \\
& \text { int }\end{array}$ & $\begin{array}{r}555 \\
\pm 154\end{array}$ & $\begin{array}{r}583 \\
\pm 164 \\
577 \\
\pm 156\end{array}$ & $\begin{array}{l}-29 \\
\pm 27 \\
-3 \\
\pm 21\end{array}$ & $\begin{array}{l}25 \\
25\end{array}$ & $\begin{array}{r}524 \\
\pm 149 \\
560 \\
\pm 158\end{array}$ & $\begin{array}{r}29 \\
\pm 27 \\
-\quad 6 \\
\pm 24\end{array}$ & $\begin{array}{l}28 \\
28\end{array}$ & $\begin{array}{r}553 \\
\pm 155 \\
558 \\
\pm 156\end{array}$ & $\begin{array}{l}-1 \\
\pm 19 \\
-4 \\
\pm 18\end{array}$ & $\begin{array}{l}24 \\
23\end{array}$ \\
\hline $\begin{array}{ll}\text { Fichier } & x^{2} \\
3 b & \text { tank }\end{array}$ & $\begin{array}{r}567 \\
\pm 156\end{array}$ & $\begin{array}{r}599 \\
\pm 167 \\
571 \\
\pm 160\end{array}$ & $\begin{array}{l}-32 \\
\pm 28 \\
-\quad 4 \\
\pm 21\end{array}$ & $\begin{array}{l}22 \\
25\end{array}$ & $\begin{array}{r}535 \\
\pm 151 \\
572 \\
\pm 158\end{array}$ & $\begin{array}{r}32 \\
\pm 28 \\
-\quad 5 \\
\pm 24\end{array}$ & $\begin{array}{l}28 \\
28\end{array}$ & $\begin{array}{r}566 \\
\pm 157 \\
571 \\
\pm 158\end{array}$ & $\begin{array}{l}-1 \\
\pm 20 \\
-5 \\
\pm 20\end{array}$ & $\begin{array}{l}24 \\
23\end{array}$ \\
\hline
\end{tabular}


duction de lait à $200 \mathrm{j}$ avec tendance à la surestimation avec un premier contrôle à la traite du matin et à la sous-estimation avec un premier contrôle à la traite du soir.

La mise en place d'un ajustement permet, quelle que soit la stratégie adoptée, de réduire le biais moyen d'estimation dans le cas de la quantité de lait, de matière protéique et du taux protéique (tableaux VI et VII). En effet, dans ce cas, on réussit à estimer correctement les productions quotidiennes. Les biais moyens observés dans le cas de la méthodologie AT30 ne différent plus selon que le premier contrôle est réalisé à la traite du matin ou du soir. Par contre, il persiste un biais non négligeable dans l'estimation du taux butyreux et de la quantité de matière grasse.

\section{BILAN - COMPARAISON DES DIFFÉ- RENTES OPTIONS D'ALLĖGEMENT}

\section{Étude de la répétabilité des mesures}

La répétabilité entre mesures est calculée par le rapport entre les composantes de variance chèvre intratroupeau et la somme de celle-ci et de la variance résiduelle.

La répétabilité des productions enregistrées entre contrôles successifs varie au cours de la campagne (tableau VIII). Pour tous les caractères (quantités et taux), elle est maximale entre contrôles du milieu de campagne. Elle est plus élevée pour la quantité de lait que pour les taux, aussi bien protéique que butyreux, intermédiaire pour les quantités de matières.

La répétabilité entre les mesures du soir et du matin est globalement plus forte que celle entre production des mois successifs. Très élevée dans le cas du taux protéique, elle est la plus faible pour le taux butyreux. Tout au long de la lactation, elle est d'un niveau comparable pour le lait et la quantité de matière protéique.

Dans l'hypothèse simplificatrice où la production totale calculée s'exprime comme la somme de $n$ mesures élémentaires de variance identique et de répétabilité (r) constantes, la perte de précision relative s'exprime par

$$
\sigma^{2}{ }^{\prime} \sigma^{2} Y=\{(n-m)(1-r)\} /\{n[1+(m-1) r]\}
$$

si l'allègement consiste à ne considérer que $m$ mesures parmi les $n$. La perte de précision est d'autant plus forte que la répétabilité entre les mesures est faible, que le nombre de mesures de référence est faible, que le nombre de contrôles supprimés est élevé. L'allègement sera donc plus dommageable avec une durée de lactation plus courte, pour les quantités de matières que pour la quantité de lait, pour le taux butyreux que pour le taux protéique.

\section{Comparaison des allègements par es- pacement des contrôles et suppression du contróle d'une des 2 traites quoti- diennes}

Cette comparaison est possible à partir des données du fichier 1 (tableau IV). Sur cet exemple, comparé au contrôle de type AT30, la perte de précision de la méthodologie A42 est nettement plus élevée que dans le cas de la méthodologie AT30. L'écart type d'erreur est réduit de moitié entre l'option A42 et l'option AT30 pour les 3 caractères quantité de lait, de matière protéique et taux protéique.

Ces tendances sont conformes aux valeurs des répétabilités entre mesures et aux résultats de différents auteurs ( $\mathrm{Mc} \mathrm{Da}$ niel, 1969; Dickinson et Mc Daniel, 1970; Putnam et Gilmore, 1970; Munro, 1976; Schaeffer et Rennie, 1976; Smith et Pear- 
Tableau VIII. Estimation des coefficients de répétabilités entre les mesures du soir et du matin d'un même contrôle et entre les contrôles mensuels successifs.

\begin{tabular}{lllllllll}
\hline$N^{\circ}$ de contrôle & 1 & 2 & 3 & 4 & 5 & 6 & 7 & 8 \\
\hline
\end{tabular}

Fichier 2

Répétabilités soir-matin

$\begin{array}{lllllllll}\text { Lait } & 0,76 & 0,78 & 0,80 & 0,82 & 0,81 & 0,83 & 0,86 & 0,86 \\ \text { QMP } & 0,73 & 0,70 & 0,77 & 0,80 & 0,80 & 0,80 & 0,83 & 0,83 \\ \text { TP } & 0,92 & 0,91 & 0,99 & 0,90 & 0,92 & 0,85 & 0,90 & 0,80 \\ \text { QMG } & 0,69 & 0,69 & 0,69 & 0,69 & 0,71 & 0,74 & 0,80 & 0,82 \\ \text { TB } & 0,64 & 0,52 & 0,54 & 0,47 & 0,54 & 0,54 & 0,63 & 0,60\end{array}$

\begin{tabular}{lccc} 
& \multicolumn{3}{c}{ Répétabilités entre contrôles } \\
Lait & $1+2+3$ & $3+4+5$ & $5+6+7+8$ \\
TP & 0,72 & 0,77 & 0,63 \\
TB & 0,64 & 0,68 & 0,62 \\
& 0,42 & 0,47 & 0,45
\end{tabular}

Fichier 3

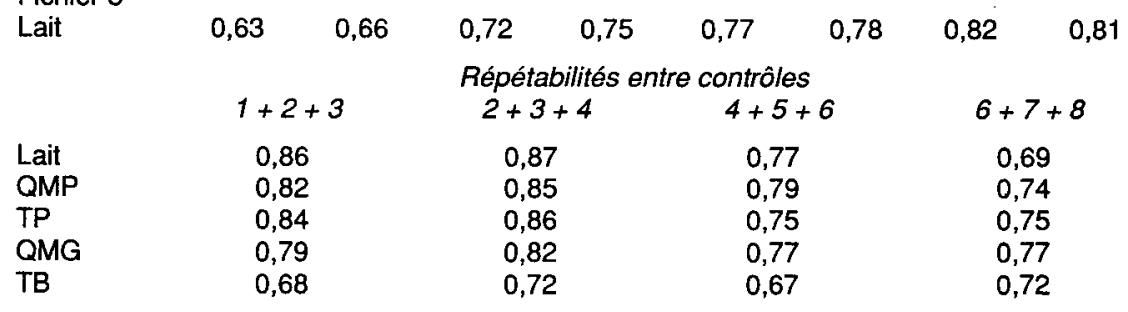

son, 1981; Hargrove et Gilbert, 1984) où le contrôle AT30 est toujours plus précis que le $A 60$ vis-à-vis de la production réelle et plus encore vis-à-vis du $\mathrm{A} 30$. Avec une fréquence plus élevée, le contrôle laitier est moins sensible à l'intervalle mise-bas premier contrôle. D'autre part, les biais importants sont moins tréquents dans le cas de la méthodologie AT30.

Pour préserver une bonne précision dans le calcul des performances de lactation, le nombre d'échantillonnages apparaît plus important que le nombre de traites mesurées à chaque contrôle. II est donc plus judicieux d'envisager un contrôle allégé respectant le rythme mensuel plutôt que d'espacer les contrôles. De plus, dans le cas du contrôle d'une seule des 2 traites, le nombre de passages du contrôleur est réduit de moitié tout en respectant un rythme mensuel, alors qu'il n'est réduit que d'un tiers dans le cas du contrôle A42. D'autre part, le maintien du rythme mensuel de passage du contrôleur semble apprécié des éleveurs.

\section{Choix d'une méthodologie basée sur le contrôle mensuel d'une des 2 traites quotidiennes et conséquences}

Le programme de sélection de l'espèce caprine s'appuie sur 2 critères de sélection : la quantité de matière et le taux protéique. 
En vue de l'adoption d'un système allégé de contrôle laitier, il importe de mesurer la perte de précision sur ces 2 critères. En raison de faibles variations du taux protéjque entre le soir et le matin, niveau de précision et importance du biais observés sur la quantité de lait peuvent être étendus aux cas de la quantité de matière et du taux protéique (tableaux IV et VI; Delorenzo et al, 1986).

Dans le cas des durées moyennes de la lactation rencontrées dans les conditions actuelles de l'élevage caprin, la stratégie AT30 permet, sans correction des performances mesurées, un calcul des performances laitières légèrement plus précis que dans le cas des stratégies AS30 et AM30. Les performances sont calculées avec un biais moyen nul.

Avec un contrôle de type AT30, pour la quantité de lait et par extension pour la quantité de matière et le taux protéique, la perte de précision est de $2,5 \%$ environ. Cette perte de précision se traduit par une réduction dans les mêmes proportions de la précision de l'estimation de la valeur génétique des reproducteurs. Pour la maintenir à son niveau actuel, il faut prévoir des informations supplémentaires, soit $2,7 \%$ de chevrettes supplémentaires par bouc mis en testage pour un caractère d'héritabilité 0,30 (cas de la QMP), soit 1 à 2 filles supplémentaires par père sur la base de 50 chevrettes de testage. Par contre, le contrôle laitier allégé se traduit par une diminution de la précision des index femelles sur performances propres (de $6,2 \%$ pour un caractère de répétabilité 0,60 ) sans possibilité de compensation. II en résulte une perte de $3 \%$ du progrès génétique potentiel à comparer à la réduction de moitié du nombre d'échantillonnages à réaliser dans chaque élevage. Dans le cas du taux butyreux et dans une moindre mesure, dans celui de la quantité de matière grasse, le contrôle laitier allégé ne permet pas un calcul de performances suffisamment précis pour être valorisé à des fins génétiques.

La stratégie AT30 introduit toutefois une hétérogénéité artificielle entre les femelles selon que le premier contrôle est réalisé à la traite du matin ou à celle du soir. Celleci se traduit par une sur- ou sousestimation des performances de lactation qui rend difficile la comparaison des femelles intra-élevage (Dickinson et Mc Daniel, 1970). Cet écart entre les 2 groupes d'animaux peut être corrigé par la mise en place d'un ajustement permettant les corrections pour les variations entre le soir et le matin (horaires de traites ou lait du troupeau le jour du contrôle). Mais cela suppose la récolte d'informations supplémentaires dont la mise en œuvre peut s'avérer difficile. La prise en compte dans les modèles d'indexation du type de traite (matin ou soir) vérifiée lors du premier contrôle de la femelle, peut être envisagée plus simplement pour maîtriser ce facteur de variation a posteriori.

Les résultats obtenus et observés dans cette étude sous-entendent la stricte application de l'alternance soir-matin à l'élevage. Une dérive dans son application entraîne une baisse de précision et une augmentation du biais moyen vers les types AM30 ou AS30 selon que les contrôles du matin ou du soir sont prépondérants. De plus, cette dérive génère un plus grand nombre (difficilement gérable) de modalités de succession des types de traites contrôlées.

Un allègement de type AT30 basé sur le contrôle d'une seule des 2 traites entraîne une baisse de la précision sur la production quotidienne. Cette information peut se révéler d'un maniement plus délicat pour le suivi des performances (Everett et Wadell, 1970a). La production à une traite est plus sensible à des variations extérieures (intervalles entre les traites, stress divers) que 
la production cumulée sur 2 traites où des compensations d'une traite sur l'autre interviennent. D'un contrôle à l'autre, les résultats sont obtenus dans des conditions différentes (traites du matin et du soir). Toutefois celle-ci reste suffisante pour les décisions techniques courantes (ajustement des rations) de l'éleveur.

\section{CONCLUSION}

II est possible de proposer la stratégie AT30 comme pratique allégée du contrôle laitier. Dans le contexte actuel de l'élevage caprin, elle est d'une efficacité compatible avec les exigences de l'indexation des reproducteurs. Relativement facile à mettre en œuvre, puisque ne nécessitant pas la récolte d'informations supplémentaires par rapport à la situation actuelle, elle a l'avantage de conserver la fréquence mensuelle de visite du contrôleur dans l'élevage. Pour sa mise en cuvre, en plus du respect de la fréquence des contrôles (identique aux normes actuellement en vigueur pour le $A 30$ ), il faut enregistrer le type de traite contrôlée (matin ou soir) pour prise en compte dans les modèles d'indexation et vérification du respect de l'alternance soir-matin. Bien que les informations ponctuelles relevées à chaque contrôle soient plus difficilement valorisables au niveau de l'élevage (difficulté pour comparer les résultats de 2 contrôles successifs puisque n'étant pas obtenus au même moment de la journée d'une fois sur l'autre), elles sont suffisantes pour le suivi technique habituel.

\section{RÉFÉRENCES}

Anderson SM, Mao IL, Gill JL (1989) Effect of frequency and spacing of sampling on accu- racy and precision of estimating total lactation milk yield and characteristics of the lactation curve. J Dairy Sci 72, 2387-2394

Barillet F (1990) Rapport du groupe du CICPLB sur le contrôle laitier des brebis. La simplification du contrôle laitier officiel de type $A$. $27^{\ominus}$ Session du CICPLP, Paris, 2-8 juillet $1990,7 \mathrm{p}$

Barillet $F$, Boichard D, Bouloc N, Gabiña D, Piacere A, Roussely M, Sigwald JP (1987) Précision et mise en œuvre dans les espèces ovines et caprines de méthodes simplifiées de contrôle laitier adaptées à leur finalité. $38^{\circ}$ Réunion annuelle de la FEZ, Lisbonne, 28 sept-1 ${ }^{\text {er }}$ oct 1987

Delorenzo MA, Wiggans GR (1986) Factors for estimating daily yield of milk, fat, and protein from a single milking for herds milked twice a day. J Dairy Sci 69, 2386-2394

Dickinson FN, Mc Daniel BT (1970) Single milking yields versus 24-hour yield for estimating lactation milk production by the test interval method. J Dairy Sci 53, 200-207

Everett RW, Wadell LM (1970a) Relationship between milking intervals and individual milk weight. J Dairy Sci 53, 548-553

Everett RW, Wadell LH (1970b) Sources of variation affecting the difference between morning and evening daily milk production. $J$ Dairy Sci 53, 1424-1429

Gabiña D, Urarte E, Arranz J (1986) Métodos de simplificacion del control lechero cuantitativo. Aplicacion a las razas ovinas del pais vasco. Invest Agrar, Prod Sanid Anim 1, 159-170

Hargrove GL, Gilbert GR (1984) Differences in morning and evening sample milkings and adjustment to daily weights and percents. $J$ Dairy Sci 67, 194-200

Lee AJ, Wardrop J (1984) Predicting daily milk yield, fat percent and protein percent from morning or afternoon tests. J Dairy Sci 67 , $351-360$

Mc Daniel BT (1969) Accuracy of sampling procedures for estimating lactation yields: a review. J Dairy Sci 52, 1742-1761

Munro GL (1976) Alternatives to monthly sampling in herd recording. Aust $J$ Exp Agric Anim Husb 16, 293-296 
Putnam DN, Gilmore HC (1970) Factors to adjust milk production to a 24 -h basis when milking intervals are unequal. J Dairy Sci 53 , 685 (abstr)

Schaeffer LR, Rennie JC (1976) AM-PM testing for estimating lactation yield. Can J Anim Sci $56,9-15$
Smith JW, Pearson RE (1981) Development and evaluation of alternate testing procedures for official records. J Dairy Sci 64,466 474

Wiggans GR (1981) Methods to estimate milk and fat yield from am/pm plans. J Dairy Sci $64,1621-1624$ 